\title{
Plant Leaf Image Detection Method Using a Midpoint Circle Algorithm for Shape-Based Feature Extraction
}

\section{B. Vijaya Lakshmi}

K.L.N. College of Engineering, Tamil Nadu, India, bviji0677@gmail.com

V. Mohan

Thiagarajar College of Engineering, Madurai, Tamil Nadu, India, vmohan@tce.edu

Follow this and additional works at: http://digitalcommons.wayne.edu/jmasm

Part of the Applied Statistics Commons, Social and Behavioral Sciences Commons, and the Statistical Theory Commons

\section{Recommended Citation}

Lakshmi, B. V. \& Mohan, V. (2017). Plant leaf image detection method using a midpoint circle algorithm for shape-based feature extraction. Journal of Modern Applied Statistical Methods, 16(1), 461-480. doi: 10.22237/jmasm/1493598420

This Regular Article is brought to you for free and open access by the Open Access Journals at DigitalCommons@WayneState. It has been accepted for inclusion in Journal of Modern Applied Statistical Methods by an authorized editor of DigitalCommons@WayneState. 


\title{
Plant Leaf Image Detection Method Using a Midpoint Circle Algorithm for Shape-Based Feature Extraction
}

\author{
B. Vijaya Lakshmi \\ K.L.N. College of Engineering \\ Tamil Nadu, India
}

\author{
V. Mohan \\ Thiagarajar College of Engineering \\ Tamil Nadu, India
}

\begin{abstract}
Shape-based feature extraction in content-based image retrieval is an important research area at present. An algorithm is presented, based on shape features, to enhance the set of features useful in a leaf identification system.
\end{abstract}

Keywords: Centroid, edge detection, Euclidean distance, feature extraction, midpoint circle

\section{Introduction}

The recognition and identification of plants permits exploration of the genetic relationship and evolutionary law of plant systems. When recognizing and identifying plants, leaf, flower, stem, fruit and other discriminating features are observed. Eventually, computers will conduct the recognition of plants automatically or semi-automatically.

Content-based retrieval methods may be classified by features such as shape, color, or texture. They are divided into subclasses by the types of algorithm used for constructing the feature vector.

Shape is an important visual feature, and it is one of the primary features for image content description. However, shape description is difficult, because it is demanding to define perceptual shape features that measure the similarity between the shapes. The problem is more complex if shape is corrupted with noise, defection, arbitrary distortion, or occlusion. Shape has been an active research area for over thirty years. In the past, shape research has been driven

B. Vijaya Lakshmi is an Assistant Professor in the Department of Master of Computer Applications. Email her at: bviji0677@gmail.com.Dr.V. Mohan is Professor and Head of the Department of Mathematics. Email him atvmohan@tce.edu. 


\section{LEAF IMAGE DETECTION USING MIDPOINT CIRCLE ALGORITHM}

mainly by object recognition. As a result, techniques of shape representation and description mostly target particular applications such as leaf classification.

The centroid radius model is frequently used as a shape feature. The centroid radius describes the length of a radius from its centroid to its boundary, and the model captures these lengths at regular intervals as shape descriptors, using the Euclidean distance. These distances are considered features for shape description.

Let $\theta$ be the regular interval (measured in degrees) between the radii. At that point, the number of intervals given by $k=(360 / \theta)$. Numbers of features depend on the fixed value of $\theta$, and this strategy cannot measure many features of the specific region. This conventional radii model generates the vector that is the normalized length of radius for shape representation. The vector depends on the order of the radii.

The purpose of this study is to propose a new shape descriptor, based on a center point and a border of the circle and contour of the leaf, which is more effective for shape description and retrieval. It overcomes the limitation of earlier descriptors by calculating radius (distance) in distinctive ways, and by upgrading the set of feature values. Distance will be calculated between the contour of the circle and contour of leaf image, rather than between the center point and border of leaves.

This descriptor leads to identification of plant leaves based on a leaf query using shape features such as contour of leaf, center point of the leaf, and border of the circle in addition to radius. The prototype of this system has been implemented and the experiment results prove the effectiveness and superiority of the proposed method.

\section{Existing Method}

\section{Centroid Radii Model (CRM)}

Tan et al. (2003) proposed the centroid radii model (CRM) for estimating shapes of objects in images. A shape is defined to be an area of black with a background of white. Each pixel is represented by its color (black or white) and its $x / y$ coordinates on the canvas. The boundary of a shape consists of a series of boundary points. A boundary point is a black pixel with at least one white pixel as its neighbor. Let $\left(x_{i}, y_{i}\right), i=1, \ldots, n$ represent the shape having $n$ boundary points. The centroid is located at the position $C\left(X_{c}, Y_{c}\right)$ which are respectively, the average of the $x$ and $y$ co-ordinates for all black pixels: 


$$
\begin{gathered}
X_{c}=\frac{\sum_{i=1}^{n} x_{i}}{n} \\
Y_{c}=\frac{\sum_{i=1}^{n} y_{i}}{n}
\end{gathered}
$$

A radius is a straight line joining the centroid to a boundary point. In the CRM, lengths of a shape's radii from its centroid to the boundary are captured as the shape descriptor at regular intervals using the Euclidean distance. More formally, let $\theta$ be the regular interval (measured in degrees) between radii (Figure $1)$. Then, the number of intervals is given by $k=(360 / \theta)$. The length $L_{i}$ of the $i^{\text {th }}$ radius formed by joining the centroid $C\left(X_{c}, Y_{c}\right)$ to the $i^{\text {th }}$ sample point $s_{i}\left(X_{i}, Y_{i}\right)$ is given by:

$$
L_{i}=\sqrt{\left(X_{c}-x_{i}\right)^{2}+\left(Y_{c}-y_{i}\right)^{2}}
$$

All radii lengths are normalized by dividing with the longest radius length from the set of radii lengths extracted. Let the individual radii lengths be $L_{1}, L_{2}, L_{3}, \ldots, L_{k}$ Where $k$ is the total number of radii drawn at an angle. If the maximum radius length is $L_{\max }$, the normalized radii lengths are given by:

$$
l_{i}=\frac{L_{i}}{L_{\max }}, i=1, \ldots, k
$$

Furthermore, without loss of generality, suppose that the intervals are taken clockwise starting from the $x$-axis direction $\left(0^{0}\right)$. Then, the shape descriptor can be represented as a vector consisting of an ordered sequence of normalized radii lengths:

$$
S=\left\{l_{0}, l_{\theta}, l_{2 \theta}, \ldots, l_{(k-1) \theta}\right\}
$$

Here, $l_{i \theta}, 0 \leq i \leq(K-1)$ is the $(i+1)^{\text {th }}$ radius from the centroid to the boundary of the shape. With sufficient number of radii, dissimilar shapes can be differentiated from each other. 

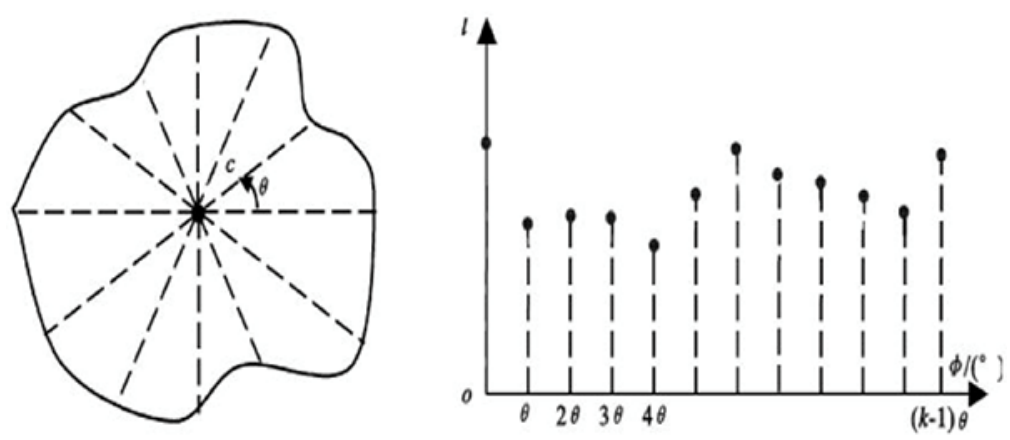

Figure 1. The centroid radii modeling of shape

\section{Midpoint Circle Algorithm}

One of the fundamental graphics primitives is the circle. The most efficient conventional algorithm for drawing a circle is the mid-point algorithm, based on Bresenham's approach (Bresenham, 1977; Ray, 2006). The mid-point algorithm starts on a quadrant boundary, and generates pixel by pixel, making either an axial or a diagonal move, depending on the sign of the decision variable.

A circle is defined as a set of points that are all at a given distance $r$ from a center positioned at $\left(X_{c}, Y_{c}\right)$. This is represented mathematically by the equation

$$
\left(x-x_{c}\right)^{2}+\left(y-y_{c}\right)^{2}=r^{2}
$$

Using equation (6), calculate the value of $y$ for each given value of $x$ as

$$
y=y_{c} \pm \sqrt{r^{2}-\left(X_{c}-X\right)^{2}}
$$

Thus, it is possible to calculate different pairs by giving step increments to $x$ and calculating the corresponding value of $y$. The midpoint circle algorithm uses an alternative approach, wherein the pixel positions along the circle are determined on the basis of incremental calculations of a decision parameter.

Let

$$
f(x, y)=\left(x-x_{c}\right)^{2}+\left(y-y_{c}\right)^{2}-r^{2}
$$


Thus, $f(x, y)=0$ represents the equation of a circle. Further, from coordinate geometry, for any point the following holds:

1. $f(x, y)=0 \rightarrow$ The point lies on the circle.

2. $f(x, y)<0 \rightarrow \quad$ The point lies within the circle.

3. $f(x, y)>0 \rightarrow$ The point lies outside the circle.

In the midpoint circle algorithm, the decision parameter at the $k^{\text {th }}$ step is the circle function evaluated using the coordinates of the midpoint of the two pixel centers, which are the next possible pixel position to be plotted.

Assume that unit increments to $x$ in the plotting process given, and the $y$ position is determined using this algorithm. Assuming that the $k^{\text {th }}$ pixel is plotted at $\left(X_{k}, Y_{k}\right)$ to determine whether the pixel at the position $\left(X_{k}+1, Y_{k}\right)$, or the one at $\left(X_{k}+1, Y_{k}-1\right)$, is closer to the circle as shown in Figure 2.

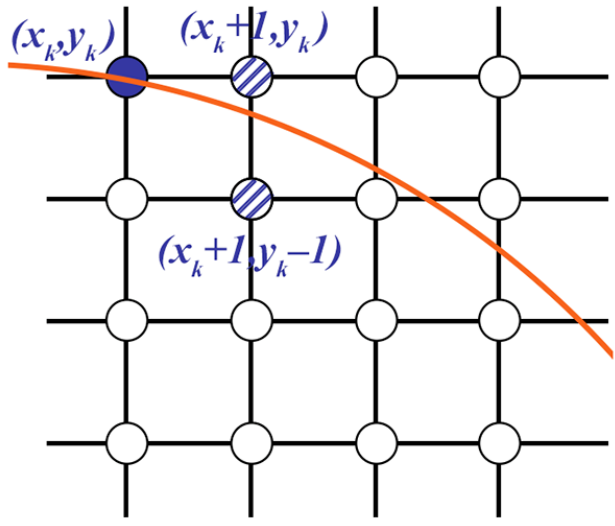

Figure 2. Plotting of the midpoint

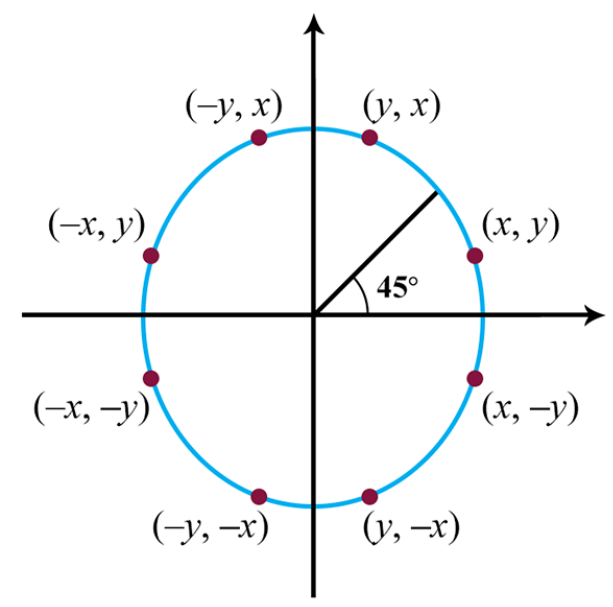

Figure 3. Eight-way symmetry

The decision parameter $p_{k}$ at the $k^{\text {th }}$ step is the circle function evaluated at the midpoint of these two pixels.

The coordinates of the midpoint of these two pixels are $\left(X_{k}+1, Y_{k}-1 / 2\right)$. Thus $p_{k}$,

$$
p_{k}=f\left(X_{k}+1, Y_{k}-\frac{1}{2}\right)=X_{k}+1^{2}+Y_{k}-\frac{1}{2}^{2}-r^{2}
$$




\section{LEAF IMAGE DETECTION USING MIDPOINT CIRCLE ALGORITHM}

Symmetry of a circle calculation of a circle point $(x, y)$ in one octant yields the circle points shown for the other seven octants, as shown in Figure 3.

The steps involved in the midpoint circle algorithm are as follows:

1. Input radius $r$ and circle center $\left(X_{c}, Y_{c}\right)$, and obtain the first point on the circumference of a circle centered on the origin as

$$
\left(X_{0}, Y_{0}\right)=(0, r)
$$

2. Calculate the initial value of the decision parameter as

$$
p_{0}=\frac{5}{4}-r
$$

3. At each $X_{k}$ position, starting at $k=0$, perform the following test: if $p_{k}<0$, the next point along the circle centered on $(0,0)$ is $\left(X_{k+1}, Y_{k}\right)$ and $p$

$$
p_{k+1}=p_{k}+2 x_{k+1}+1
$$

otherwise, the next point along the circle is $\left(X_{k}+1, Y_{k}-1\right)$. And

$$
p_{k+1}=p_{k}+2 x_{k+1}+1-2 y_{k+1}
$$

where

$$
2 x_{k+1}=2 x_{k}-2 \text { and } 2 y_{k+1}=2 y_{k}-2 \text {. }
$$

4. Determine symmetry points in the other seven octants.

5. Move each calculated pixel position $(x, y)$ onto the circular path centered on $\left(X_{c}, Y_{c}\right)$ and plot the coordinate values.

$$
x=x+x_{c}, y=y+y_{c}
$$

6. Repeat steps 3 through 5 until $x \geq y$.

By using this algorithm, a circle will be formed in a two-dimensional plane. 


\section{Related Works}

Chaki and Parekh (2011) proposed an approach that consists of comparing binary versions of the leaf images through superposition and using the sum of nonzero pixel values of the resultant as the feature vector. Consider the invariant (M-I) model and centroid radii (C-R) model. In M-I, central moments and normalized central moments are calculated. In $\mathrm{C}-\mathrm{R}$, the length of a shape's radii from its centroid of the boundary is captured at regular intervals as the shape descriptor using Euclidean distance. In the method, two images are in binary superposition; a large-value sum would indicate high similarity between images, and a small-value sum would indicate low similarity between images. A comparison of the recognition accuracy of these two methods with a proposed method used 180 leaf images from the plantScan database, three classes of 60 samples each. From that set, 120 images were used for training and 60 images were tested.

Lee and Hong (2013) proposed a leaf recognition system for plant classification, employing major vein, frequency domain data by using fast Fourier transformation. Dilation and erosion operations were used to extract leaf veins, and a projection histogram was calculated for both horizontal and vertical directions in order to measure the vein distribution of the leaf. The authors extracted 10 features, using fast Fourier transformation, distance, and phase; another 10 features were extracted using leaf length, width, area, and perimeter; and a final feature, convex hull, was also extracted. This system was applied to 1970 leaf images, consisting of 32 types of leaves with 50 to 77 samples each and implemented in VC++ 6.0, Intel OpenCV Library. The accuracy of this method was reported at $97.19 \%$.

Deokar, Zope, and Suralkar (2013) presented feature point extraction. The feature points were extracted from a leaf image based on the geometric center. The authors proposed two schemes, vertical and horizontal, to extract feature points. In the vertical scheme, leaf images are split vertically in half with respect to a central point. Each half is then split horizontally in a number of repetitions, until 14 feature points are obtained. This process is repeated starting on the leaf's horizontal access, obtaining 14 more feature points for a total of 28 .

Fulsoundar, Kadlag, Bhadale, Bharvirkar \& Godse (2014) created an Android application to identify plant species based on photographs of the plant's leaves taken with a mobile phone. At the heart of this application is an algorithm that acquires morphological features of the leaves, computes well-documented metrics consisting of the angle code histogram $(\mathrm{ACH})$, and classifies the species. The first algorithm was prepared against several samples of known plant species 


\section{LEAF IMAGE DETECTION USING MIDPOINT CIRCLE ALGORITHM}

after being used to classify unknown query species. Supported by features designed into the application such as touch screen image rotation and contour preview, the algorithm has been very successful in properly classifying species inside the training library.

Bong, M. F., Sulong, G., Kumoi, R., \& Rahim, M. S. M. (2015) proposed a novel approach to cluster the species of plants based on their lobes, sinuses and margin. Firstly, all the boundary points in a clockwise or anticlockwise direction were selected. Then, a center point for leaf boundary points was estimated, and used to compute the distance between the leaf boundary points and the center. Next, the peaks and valleys from the computed distance were located, where peaks represent lobes and valleys represent the sinuses. The number of peaks and valleys was calculated to cluster the plant, according to a rule-based method. The accuracy of this method for plant clustering is up to 100 percent.

\section{The Proposed Circle-based Radii Model (CBRM)}

A new Circle-based Radii Model is now proposed for shape descriptor. A circle is formed by using a midpoint circle algorithm based on the center point of the leaf, as discussed earlier under that heading. Here, the radius is 0.5 , after forming the circle; the proposed method uses two phases to obtain four feature points on the circle of a leaf image in the two-dimensional plane.

\section{Architecture of the Circle-based Radii Model}

The architecture of proposed circle-based radii model for the plant leaf recognition of $2 \mathrm{D}$ objects is given in the Figure 4. 


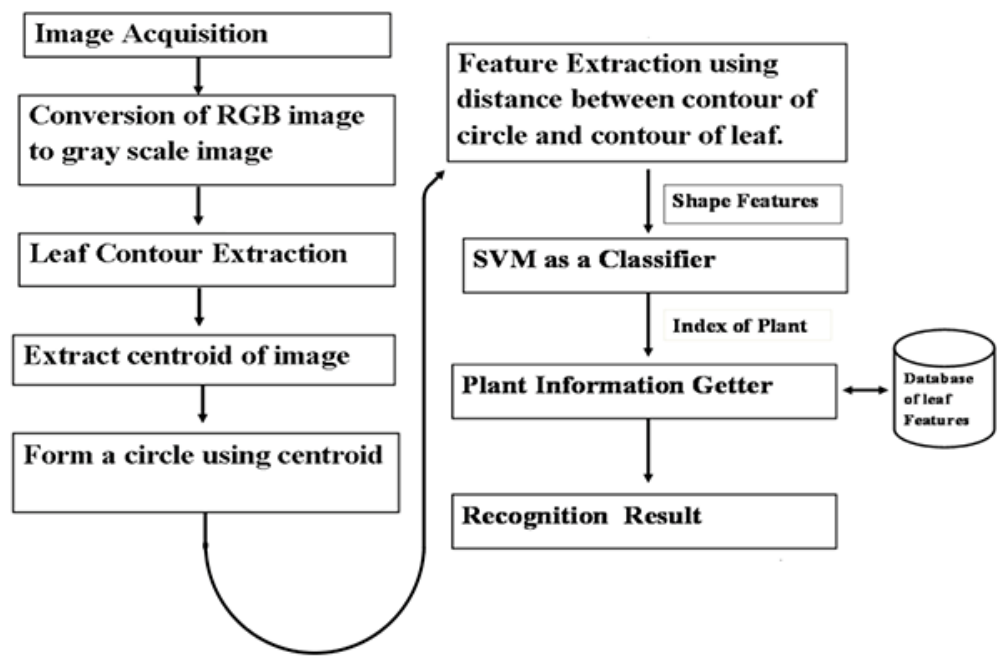

Figure 4. Architecture for leaf recognition system

It includes the following stages: preprocessing, circle formation and feature extraction.

\section{Preprocessing}

The leaf images may be acquired utilizing an advanced digital camera. There is no confinement on image resolution or format. Resulting digital images are usually in RGB color space; some may be grayscale. The fundamental objective of preprocessing is to a) identify the leaf in an image and b) discard all information other than the leaf shape. The initial step is to resize all the leaves to $256 \times 256$, then convert any RGB image to grayscale. Below is the equation used to convert RGB value of a pixel to its grayscale value.

$$
\text { Gray }=0.2989 * \mathrm{R}+0.5870 * \mathrm{G}+0.1140 * \mathrm{~B}
$$

where R, G, B corresponds to color of the pixel.

Finally, the Sobel edge detection method is applied to the resulting image. The entire procedure is shown in Figure 5. 


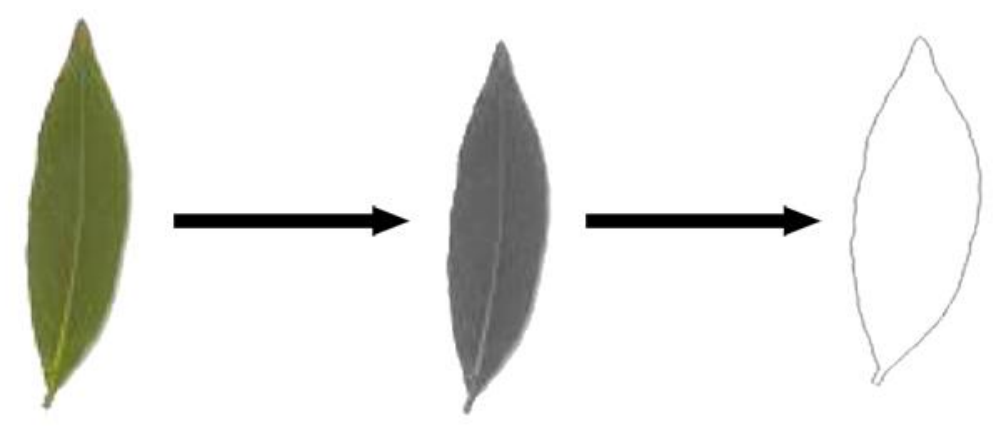

Figure 5. Sample of color to gray conversion and contour extraction of Leaf

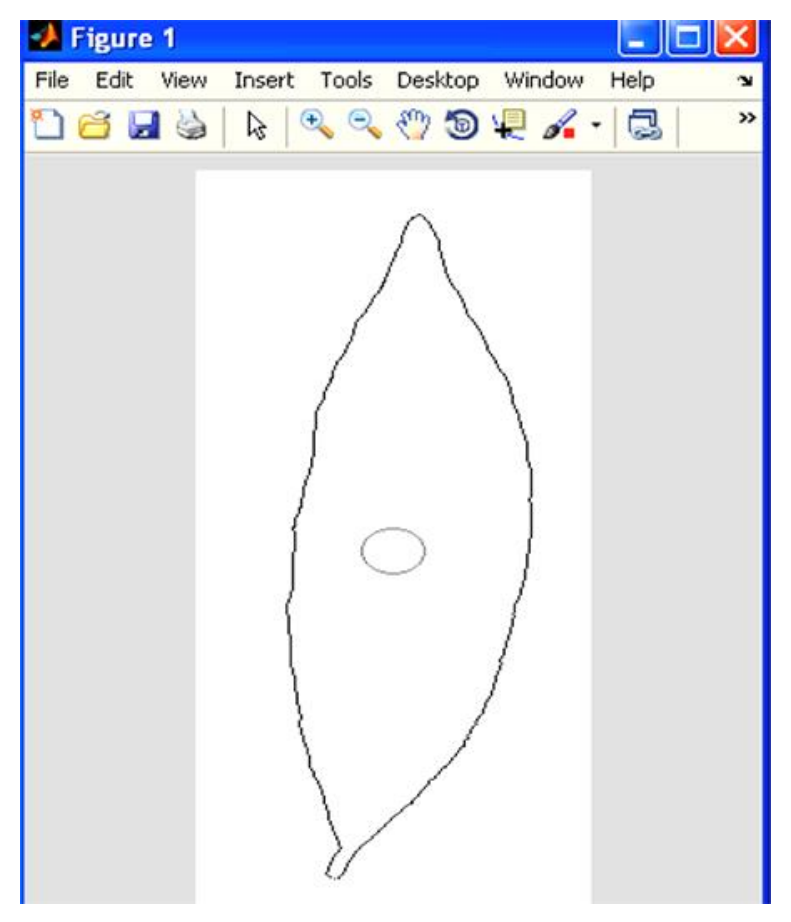

Figure 6. Circle formation within leaf

\section{Circle Formation inside the Leaf}

Once the contour of the leaf image is detected, a circle is formed based on the center point of the leaf image by using the midpoint circle algorithm. In the midpoint circle algorithm, an eight-way symmetry sample is used as in Figure 3. 
The midpoint circle algorithm works on the same midpoint concept as Bresenham's line algorithm (Bresenham, 1977). Figure 6 shows the circle arrangement inside the leaf image.

\section{Feature Extraction}

The proposed techniques of the circle-based radii model (CBRM) are discussed here. Subsequent to forming the circle, the method obtains four feature points on the circle of a leaf image in the two-dimensional plane. The distance is calculated between the contour of the circle and the contour of the leaf image as shown in Figure 7. They are described as:

The distance between the contour of the circle $\left(x_{i}, y_{i}\right)$ and the contour of the leaf image $\left(x_{r}, y_{r}\right)$ is called Realdistance, and is shown in Figure 7.

The distance between a point in a vertical or horizontal line running through the center point $\left(x_{c}, y_{c}\right)$ and contour of circle $\left(x_{i}, y_{i}\right)$ is called Imaginarydistance.

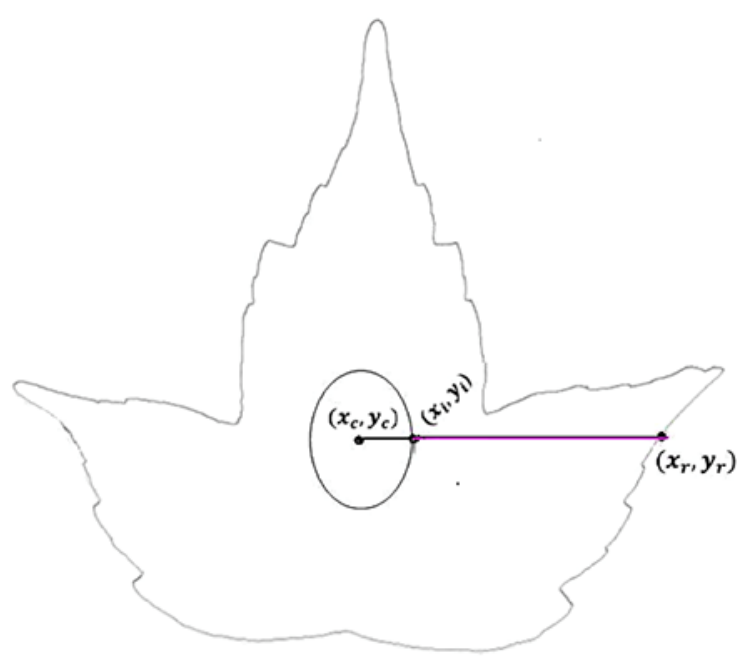

Figure 7. Circle-based radii model (CBRM)

In the proposed method, the Realdistance (RD) are calculated using equation (15) for feature extraction:

$$
R D_{i}=\sqrt{\left(X_{i}-x_{r}\right)^{2}+\left(Y_{i}-y_{r}\right)^{2}}
$$




\section{LEAF IMAGE DETECTION USING MIDPOINT CIRCLE ALGORITHM}

In the first phase, splitting the circle vertically through the center derives its top and bottom points. Then, left-to-right row-wise scanning from the top point of the circle to the leaf contour is conducted to the bottom point, as illustrated in Figure 8(a). Euclidean distance is calculated between the contour of the circle and contour of the leaf image, rather than between the center point and border of leaves. This set of Realdistance values is considered the first set of feature points for shape description. The process is repeated in the opposite direction, as shown in Figure 8(b), resulting in the second set of shape descriptor features.

The second phase repeats this process, splitting the circle horizontally and measuring similar distances vertically, to derive the third and fourth set of shape descriptor features, as illustrated in Figure 8(c) and 8(d).

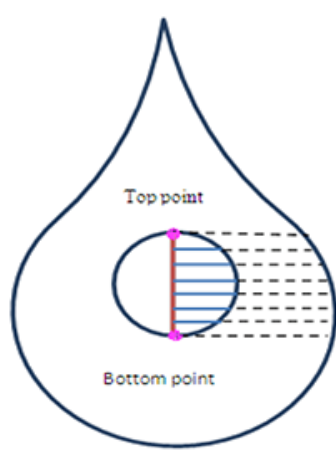

(a)

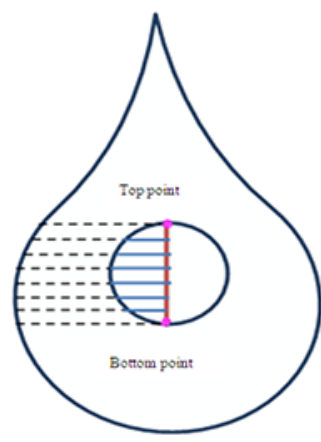

(b)

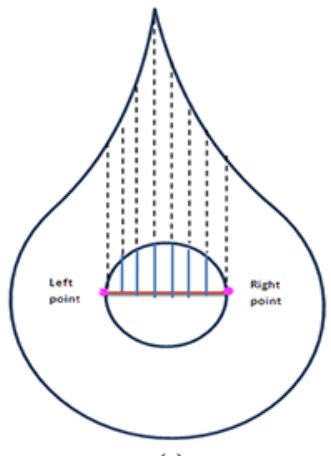

(c)

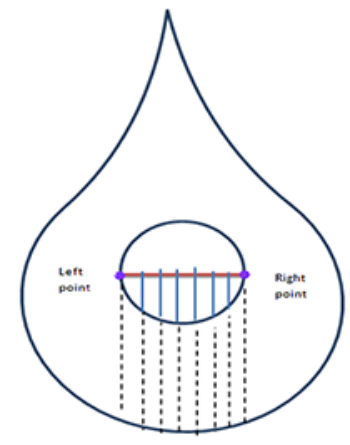

(d)

Figure 8. Real distance calculation (a) Right direction (b) Left direction (c) Top direction (d) Down direction

\section{Algorithm: Circle-based Radii Model}

The CBRM algorithm for radius calculation is summarized in the following steps.

Input: Image and radius

Output: $N$ number of features

Step 1: Load the image and detect the border of the image.

Step 2: Assign image size as $[a, b]$ 
Step 3: Find the center point of the image $\left(x_{c}, y_{c}\right)$

Step 4: Draw the circle by using Midpoint circle algorithm.

Assign:

$\begin{array}{lll}\text { Center point } & = & \left(x_{c}, y_{c}\right) \\ \text { Top point } & = & \left(x_{c}-\text { radius }, y_{c}\right) \\ \text { Bottom point } & = & \left(x_{c}+\text { radius }, y_{c}\right) \\ \text { Leaf point } & = & \left(x_{c}, y_{c}-\text { radius }\right) \\ \text { Right Point } & = & \left(x_{c}, y_{c}+\text { radius }\right) \\ \text { Count }=0, \text { Flag }=0 & \end{array}$

The following steps are used to extract the features by vertical splitting of the circle

Step 1: Divide the circle in half vertically from the top point to the bottom point.

Apply steps 2 to 7 to right half of the circle.

Step 2: Start scanning from top point to the bottom point in a row-wise manner.

Step 3: Take the every column value and check the following conditions one by one.

Step 4: Check image $(i, j)$ is equal to the border of the circle or not. If it is bordered, assign Flag $=1$.

Step 5: Check flag $>0$ (zero), get the value of $\left(x_{i}, y_{i}\right)$ coordinate

Step 6: Check image $(i, j)$ is equal to border of the image. If it is

i. Get the value of $\left(x_{r}, y_{r}\right)$ coordinate

ii. Calculate the distance using equation (15)

iii. $\quad$ Reassign Flag $=0$ (zero) 


\section{LEAF IMAGE DETECTION USING MIDPOINT CIRCLE ALGORITHM}

Step 7: Go to the next row, repeat the steps from 4 to 7 until the bottom point of the circle. These are considered the first set of features for shape description

Step 8: Repeat the steps 2 to 7 to the left half side of the circle. This process will produce the second set of feature values.

The following steps are used to extract sets three and four:

Step 10: Divide the circle in half horizontally from the leftmost point to the rightmost point.

Step 11: Start reading from the left point to the right point in column wise manner.

Step 12: Take the every row value of each column and check the following conditions one by one, as in steps 4 to 6 .

Step 13: Go to the next column, repeat the steps 4 to 6 until right point of the circle.

Step 14: Repeat the steps 4 to 6 to bottom half side of the circle.

Calculating all distances in four directions in this way, the number of features and sets of feature values will be obtained.

\section{Experiments and Results}

Plant leaves classification framework is developed and executed in MATLABR2009b, to test the retrieval effectiveness and performance of the CBRM. To evaluate the effectiveness of the proposed approach, recognition procedure is carried out on a large texture dataset, provided by the Intelligent Computing Laboratory at the Chinese Academy of Science (ICL CAS, n.d.). Samples of leaf images belonging to various classes are shown in Figure 9. A sample converted gray scale leaf image is shown in Figure 10.

Experiments are performed by using 50 of 220 classes from the ICL dataset. Fifteen leaves are selected randomly from each class. 750 total $(15 \times 50$ classes $)$ sample leaves are trained. In conventional centroid radii model, twelve features 
are extracted when $\theta=30^{\circ}$. Using the proposed method, 11 features are extracted from each direction, for a total of 44 . Extracted feature values are stored in feature vector. In the end, the dataset generates 33,000 (750 leaves $\times 44$ features) feature values.

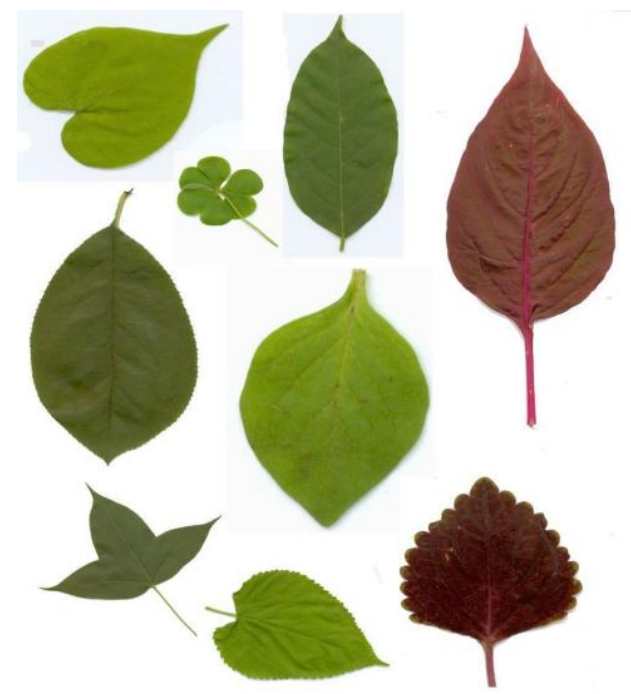

Figure 9. A sample of plant leaf images taken from the ICL plant leaf dataset

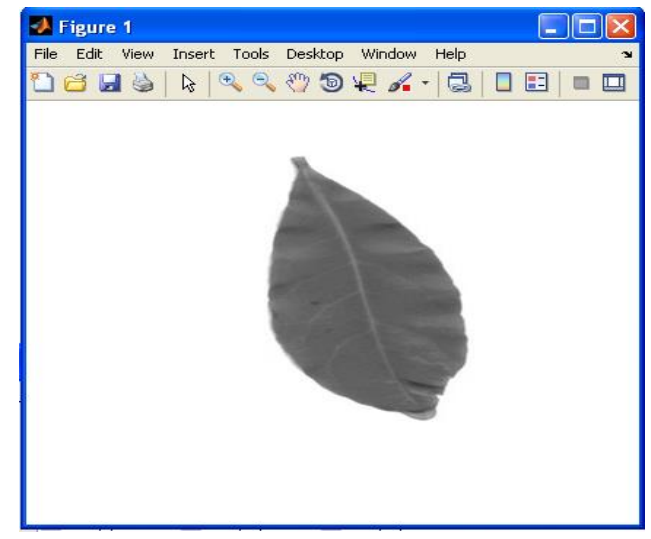

Figure 10. Transformed gray scale leaf image

Presented in Figure 11 are the results obtained with CBRM. In the testing phase, 15,000 leaves were tested randomly for the accuracy of the proposed technique. The recognition process is carried out by comparing the length of the tested circle radii with the reference. The input query image feature vector is compared with trained feature set using SVM classifier. Average of recognition of each class is given in Table 1. The performance comparison of the proposed CBRM with the centroid radii model is shown in Figure 12. The accuracy rate of the proposed method is shown to be $93.33 \%$. 


\section{LEAF IMAGE DETECTION USING MIDPOINT CIRCLE ALGORITHM}

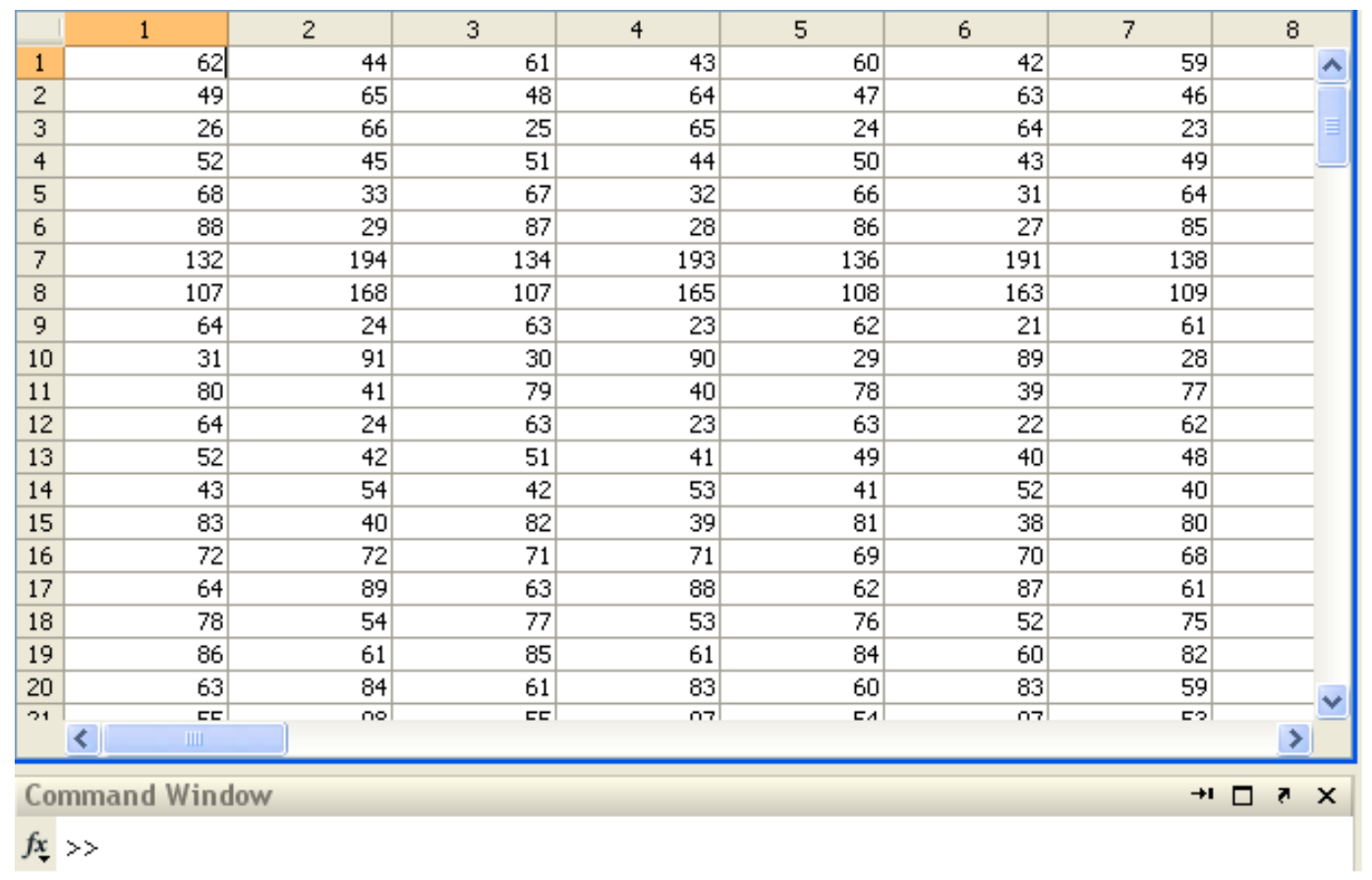

Figure 11. Set of feature value of trained leaves

Table 1. CBRM recognition accuracy for each class of leaf

\begin{tabular}{|c|c|c|c|c|c|c|c|c|c|}
\hline Class & Accuracy & Class & Accuracy & Class & Accuracy & Class & Accuracy & Class & Accuracy \\
\hline 1 & $90 \%$ & 11 & $95 \%$ & 21 & $89 \%$ & 31 & $94 \%$ & 41 & $88 \%$ \\
\hline 2 & $92 \%$ & 12 & $88 \%$ & 22 & $89 \%$ & 32 & $93 \%$ & 42 & $87 \%$ \\
\hline 3 & $94 \%$ & 13 & $84 \%$ & 23 & $92 \%$ & 33 & $95 \%$ & 43 & $96 \%$ \\
\hline 4 & $90 \%$ & 14 & $94 \%$ & 24 & $92 \%$ & 34 & $94 \%$ & 44 & $95 \%$ \\
\hline 5 & $90 \%$ & 15 & $93 \%$ & 25 & $94 \%$ & 35 & $84 \%$ & 45 & $97 \%$ \\
\hline 6 & $89 \%$ & 16 & $91 \%$ & 26 & $97 \%$ & 36 & $96 \%$ & 46 & $93 \%$ \\
\hline 7 & $86 \%$ & 17 & $97 \%$ & 27 & $86 \%$ & 37 & $95 \%$ & 47 & $92 \%$ \\
\hline 8 & $92 \%$ & 18 & $88 \%$ & 28 & $95 \%$ & 38 & $94 \%$ & 48 & $96 \%$ \\
\hline 9 & $86 \%$ & 19 & $86 \%$ & 29 & $89 \%$ & 39 & $93 \%$ & 49 & $96 \%$ \\
\hline 10 & $84 \%$ & 20 & $84 \%$ & 30 & $89 \%$ & 40 & $89 \%$ & 50 & $93 \%$ \\
\hline
\end{tabular}




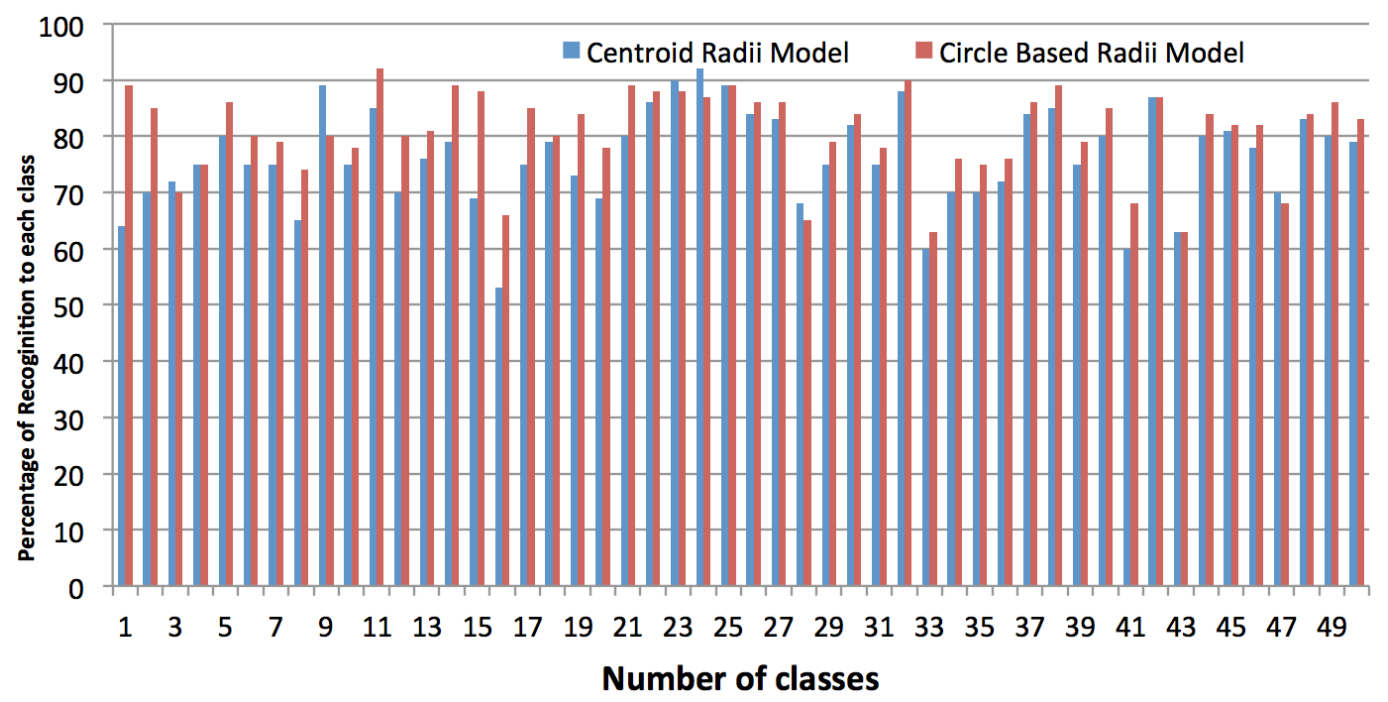

Figure 12. Performance comparison graph of the proposed circle-based radii model with the centroid radii model

Shown in Figure 13 is the 3D view-confusion matrix of the proposed circlebased radii model. Shown in Figure 14 is the 3D view of positive classification rate of the circle-based radii model. Shown in Figure 15 is the accuracy of the proposed circle-based Radii model.

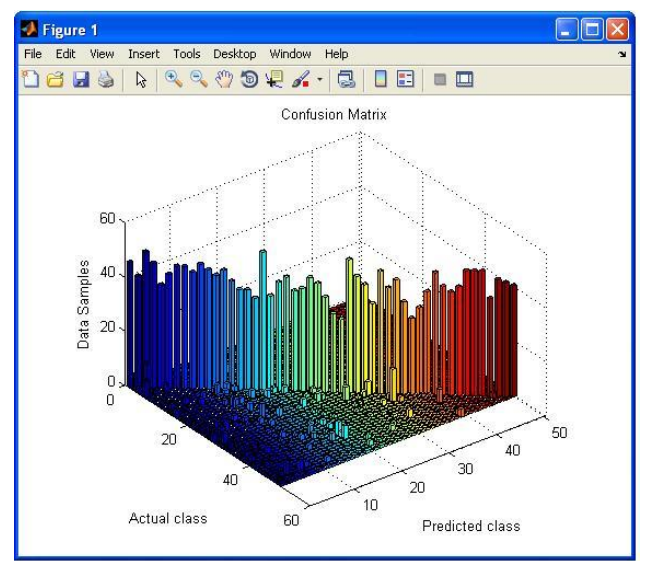

Figure 13. 3D view-confusion matrix of circle based radii model

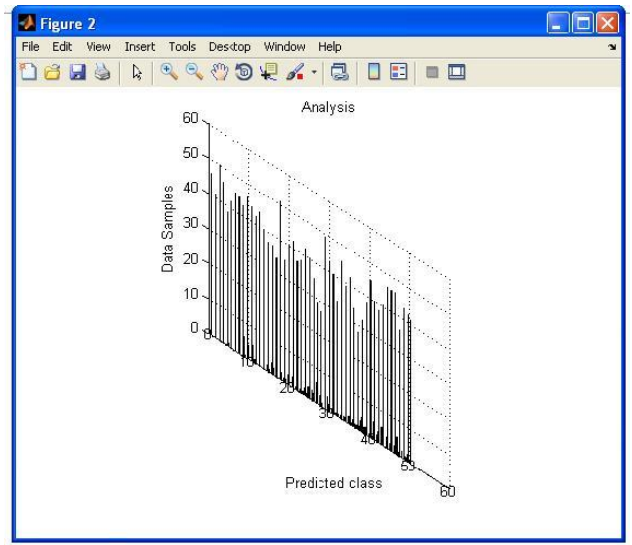

Figure 14. 3D view of positive classification rate of circle-based radii model 


\section{LEAF IMAGE DETECTION USING MIDPOINT CIRCLE ALGORITHM}

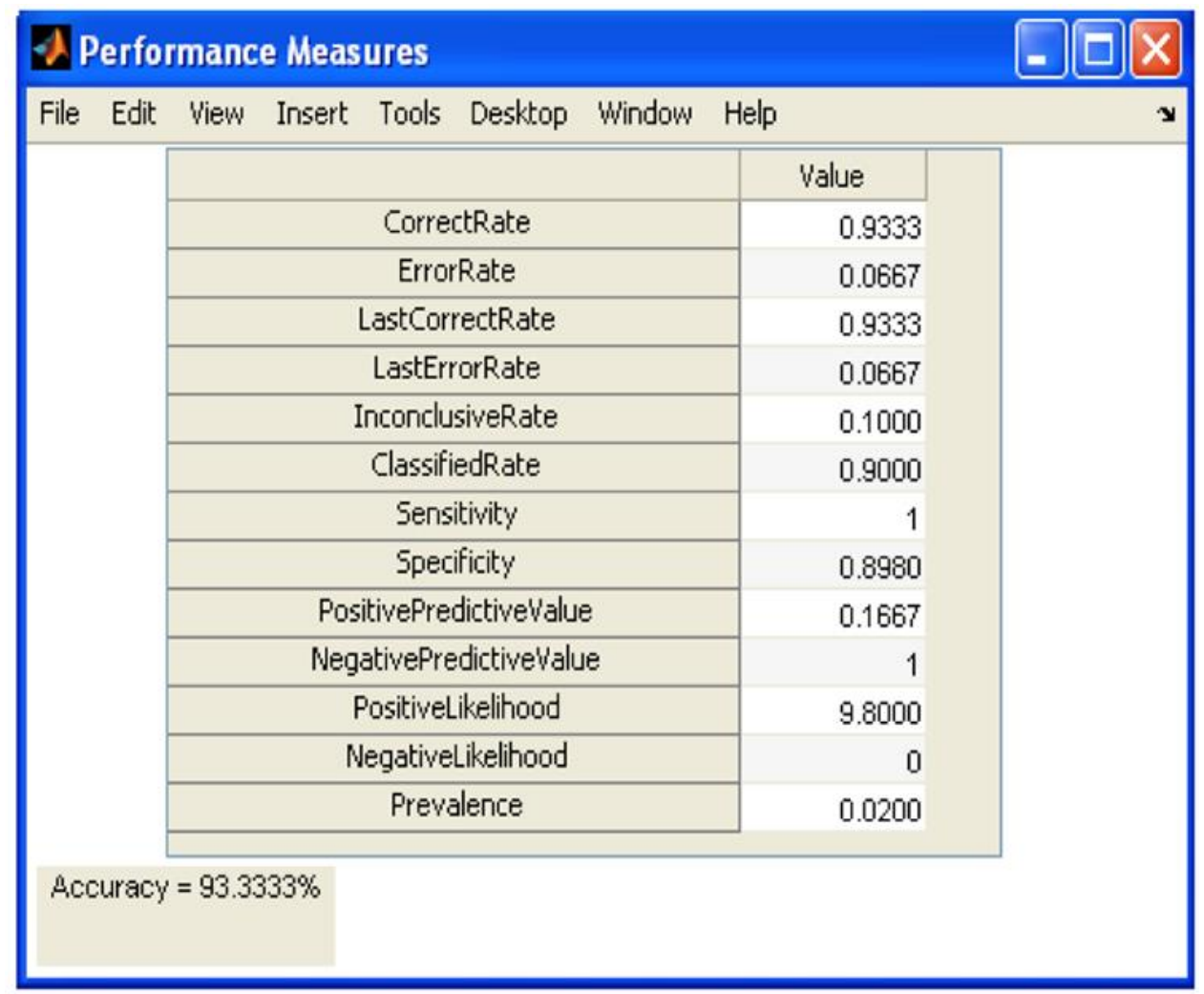

Figure 15. Accuracy of proposed circle-based radii model

\section{Conclusion}

One of the most important low-level features in content-based image retrieval is the shape. In this study a new shape descriptor, the circle-based radii model, was proposed. It is based on the center point and border of a circle centered inside the contour of a leaf. It is a successful feature extraction technique for the plant leaf classification system.

In the conventional method, the centroid radii model, the lengths of the radii from the centroid to the boundary are used to represent the shape, and the angular interval between radii is fixed. This conventional radii model generates a vector that is the normalized length of radii for shape representation. The vector depends on the order of the radius.

The proposed shape descriptor differs from the conventional centroid radii model in such a way that the distance will be calculated between the contour of 
the circle and contour of leaf image, instead of calculating distance between the center point and border of the leaf. In this method, 44 features are extracted from every leaf in specified regions and compared with standard database features of the trained leaf images by using the SVM classifier.

By using the proposed method, the plant has been identified successfully in a large number of classes. The proposed method upgrades the set of feature values. The experimental results indicate that the proposed method shows significant improvement in terms of the increased number of features, and enhances the feature value. Accuracy of proposed circle-based radii model for the shape descriptor is $93.33 \%$ (the centroid radii model achieves $85.92 \%$ accuracy), indicating that the circle-based radii model is more suitable for a plant leaf classification system given its high retrieval performance.

\section{References}

Bong, M. F., Sulong, G., Kumoi, R. \& Rahim, M. S. M. (2015). Classify the plant species based on lobes, sinuses and margin. Jurnal Teknologi, 75(2), 27-31. doi: 10.11113/jt.v75.4973

Bresenham, J. E. (1977). A linear algorithm for incremental digital display of circular arcs. Communications of the ACM, 20(2), 100-106. doi:

10.1145/359423.359432

Chaki, J. \& Parekh, R. (2011). Plant leaf recognition using shape based features and network classifiers. International Journal of Advanced Computer Science and Applications, 2(10). doi: 10.14569/ijacsa.2011.021007

Deokar, S. R., Zope, P. H., \& Suralkar, S. R. (2013). Leaf recognition using feature point extraction and artificial neural network. International Journal of Engineering Research and Technology, 2(1).

Fulsoundar, K., Kadlag, T., Bhadale, S., Bharvirkar, P. \& Godse, S. P. (2014). Detection and classification of plant leaf diseases. International Journal of Engineering Research and General Science, 2(6), 868-874.

Intelligent Computing Laboratory, Chinese Academy of Science. (n.d.) Intelligent Computing Laboratory (ICL) data set [data set].

Lee, K. B. \& Hong, K. S. (2013). An implementation of leaf recognition system using leaf vein and shape. International Journal of Bio-Science and BioTechnology, 5(2), 57-66. 


\section{LEAF IMAGE DETECTION USING MIDPOINT CIRCLE ALGORITHM}

Ray, B. K. (2006). An alternative approach to circle drawing. Journal of the Indian Institute of Science, 86, 617-623.

Tan, K. L., Ooi, B. C. \& Thiang, L. F. (2003). Retrieving similar shapes effectively and efficiently. Multimedia Tools and Applications, 19(2), 111-134. doi: 10.1023/a:1022142527536 